\title{
Pineal cyst resection in the absence of ventriculomegaly or Parinaud's syndrome: clinical outcomes and implications for patient selection
}

\author{
M. Yashar S. Kalani, MD, PhD, ${ }^{1,2}$ David A. Wilson, MD, ${ }^{1}$ Nicolas Olmo Koechlin, MD, ${ }^{2}$ \\ Hazem J. Abuhusain, MBBCh, PhD, ${ }^{2}$ Brian J. Dlouhy, MD, ${ }^{2,3}$ Manuri P. Gunawardena, \\ Kyoko Nozue-Okada, MD, ${ }^{2}$ and Charles Teo, MBBS, FRACS ${ }^{2}$ \\ ${ }^{1}$ Division of Neurological Surgery, Barrow Neurological Institute, St. Joseph's Hospital and Medical Center, Phoenix,
Arizona; ${ }^{2}$ Centre for Minimally Invasive Neurosurgery, Prince of Wales Hospital, Sydney, New South Wales,
Australia; and ${ }^{3}$ Department of Neurosurgery, University of lowa Hospitals and Clinics, lowa City, lowa
}

OBJECT Surgical indications for patients with pineal cysts are controversial. While the majority of patients harboring a pineal cyst require no treatment, surgery is a well-accepted option for a subset of those patients with secondary hydrocephalus or Parinaud's syndrome. The majority of pineal cysts are identified incidentally during workup for other potential conditions, which may or may not be related to the presence of the cyst. In the absence of clear obstruction of CSF pathways, the treatment of presumed symptomatic pineal cysts is debatable. To clarify the role of surgery in these borderline cases, the authors reviewed their experience with resection of pineal cysts in the absence of ventriculomegaly or Parinaud's syndrome.

METHODS The authors retrospectively reviewed medical records and imaging of all patients surgically treated between 2001 and 2014 with a pineal cyst in the absence of ventriculomegaly and Parinaud's syndrome. The presenting symptoms, preoperative cyst size, preoperative radiographic aqueductal compression, extent of resection, and radiographic and clinical follow-up were documented.

RESULTS Eighteen patients (14 female and 4 male; mean age 24 years, range 4-47 years) underwent cyst resection in the absence of ventriculomegaly or Parinaud's syndrome. Presenting symptoms included headache (17 patients), visual disturbances (10 patients), gait instability (5 patients), dizziness (5 patients), episodic loss of consciousness (2 patients), and hypersomnolence (1 patient). The mean preoperative cyst diameter was $1.5 \mathrm{~cm}$ (range 0.9-2.2 cm). All patients had a complete resection. At a mean clinical follow-up of 19.1 months (range postoperative to 71 months), 17 (94\%) patients had resolution or improvement of their presenting symptoms.

CONCLUSIONS The authors' results suggest that ventriculomegaly and Parinaud's syndrome are not absolute requisites for a pineal cyst to be symptomatic. Analogous to colloid cysts of the third ventricle, intermittent occlusion of cerebrospinal fluid pathways may cause small pineal cysts to become intermittently symptomatic. A select cohort of patients with pineal cysts may benefit from surgery despite a lack of hydrocephalus or other obvious compressive pathology.

http://thejns.org/doi/abs/10.3171/2014.9.JNS141081

KEY WORDS pineal cyst; symptomatic; occipital-transtentorial approach; surgery; oncology

$\mathrm{B}$ ENIGN pineal cysts are common in both the pediatric and adult populations. Magnetic resonance imaging studies estimate a $1 \%-2 \%$ overall population prevalence, ${ }^{3,8,12}$ while autopsy studies suggest that up to $40 \%$ of the general population harbors a pineal cyst. ${ }^{14,24}$ Most pineal cysts are asymptomatic, have a benign natural history, and, in the era of modern neuroimaging, are increasingly discovered incidentally. $2,8,12$
The high prevalence of pineal cysts coupled with the nonspecific symptoms with which these patients may present, particularly headache, often prevents the determination of causation from being made. ${ }^{3,8,9,23}$ Consequently, the management of patients with neurological symptoms and a pineal cyst in the absence of hydrocephalus is not well defined. Some authors suggest that, in the absence of obstructive hydrocephalus or clear signs of tectal com- 
pression, symptoms should not be attributed to the pineal cyst and these patients should be managed conservatively. ${ }^{3}$ Other sporadic reports suggest that a pineal cyst may cause intermittent obstruction of the sylvian aqueduct or compression of critical neural structures, making resection a reasonable option. ${ }^{7,13,15,17-21,25,26}$

Noncommunicating hydrocephalus and Parinaud's syndrome are rarely caused by a simple pineal cyst. Therefore, the benefit of surgery in the absence of intracranial hypertension is unclear. ${ }^{1-3,11,13}$ To define surgical indications, we reviewed our experience with pineal cysts presenting in the absence of ventriculomegaly or Parinaud's syndrome treated with minimally invasive surgery.

\section{Methods \\ Patients}

We retrospectively reviewed medical records and radiographic imaging of all patients who presented with a pineal cyst to the Centre for Minimally Invasive Neurosurgery (Sydney, Australia) between 2001 and 2014. We retrospectively reviewed patient age, sex, presenting symptoms, symptom duration, preoperative cyst size (maximum diameter), presence of ventriculomegaly on preoperative MRI, presence of Parinaud's syndrome on presentation, surgical approach, and perioperative complications. Only patients without radiographic ventriculomegaly or Parinaud's syndrome on presentation were included in this study.

\section{Selection for Surgery}

The decision to offer surgery was guided by experience with colloid cysts of the third ventricle, for which ample literature supports the notion of intermittent CSF pathway occlusion resulting in symptoms.

In all cases, patients underwent a thorough medical workup and presumptive treatment of their symptoms (including headaches and vertigo) by a neurologist. Symptoms deemed suspicious included 1) headaches that either had an intracranial pressure character or were positional; 2) headaches that were associated with another symptom of intracranial hypertension such as nausea, vomiting, drowsiness and visual disturbance; 3 ) other symptoms that were suggestive of compression in the peri-pineal region such as visual or sensory dysfunction; 4) visual obscuration of any degree; 5) intermittent changes in level of consciousness with spontaneous recovery that may or may not have been initiated by a change in head posture; and 6) midsagittal MRI that demonstrated compression of the underlying aqueduct. Typical migraine headaches, including clear nonpositional triggers and associated photosensitivity and auras, were deemed nonindicative of CSF obstruction, and surgery was not offered to patients with these indications.

In some cases, differentiating between intermittent CSF obstruction and other causes of headache was not possible, and the decision to proceed with surgery depended on multiple factors, including cyst size, presence of aqueduct compression on imaging, and intractability of symptoms. Other less common indications for offering surgery included the presence of inexplicable neurological symptoms for which no cause could be found and that, in theory, may have been due to intermittent CSF obstruction or pineal dysfunction. This category included 2 patients with episodic loss of consciousness with workups negative for epilepsy or cardiac dysfunction and 1 patient with a clearly documented hypersomnolence syndrome for which no cause could be found. In all cases in which surgery was offered, patients were clearly informed before surgery that a definitive attribution of symptoms to the pineal cyst was not possible and that they may gain no benefit from surgery while being exposed to risks as a result of intervention.

\section{Clinical Outcomes}

Patients were independently assessed by 3 neurosurgeons 6 weeks following surgery and at last follow-up, including an examiner who was not the senior surgeon (C.T.). Clinical parameters including visual dysfunction, Parinaud's syndrome, headache, nausea, vomiting, motor strength, need for CSF shunt, and need for reoperation were evaluated and compared with the preoperative baseline.

\section{Radiographic Outcomes}

Patients underwent MRI at 6 weeks following surgery and then at various time points thereafter based on the discretion of the treating neurosurgeon. In each case the last available MRI was reviewed and the presence of any residual or recurrent cyst was noted.

\section{Results \\ Patients}

During the study period, 86 patients presented with presumed symptomatic pineal cysts in the absence of ventriculomegaly or Parinaud's syndrome. Of these, 18 patients were deemed surgical candidates based on history and examination findings; these patients underwent resection. The mean patient age was 24 years (range 4-47 years). The female/male ratio was 3.5:1. All patients were offered surgery on the basis that the presenting symptoms were likely due to intermittent CSF obstruction or local mass effect. The presenting symptoms included headaches $(n=17)$, visual disturbances $(n=10)$, gait disturbance $(n$ $=5)$, dizziness $(n=5)$, episodic loss of consciousness $(n=$ $2)$, and hypersomnolence $(n=1)$. Headache was usually intermittent, often positional, and occasionally associated with bilateral visual blurring and diplopia.

\section{Imaging Characteristics}

The mean maximum cyst diameter was $1.5 \mathrm{~cm}$ (range $0.9-2.2 \mathrm{~cm})$. No patients demonstrated radiographic ventriculomegaly.

\section{Surgical Approach and Complications}

All patients underwent a supracerebellar, infratentorial or an occipital, interhemispheric, transtentorial approach for complete macroscopic resection. All specimens were pathologically confirmed to be simple pineal cysts. Twelve of the 18 patients experienced transient disconjugate eye movements for varying periods of time postoperatively. All of these patients had complete resolution at last followup. One patient underwent a supratentorial approach and 
complained of a left homonymous hemianopia 3 days after surgery. She had complete resolution within 2 weeks. One patient suffered a temporary facial droop. There were no instances of infection or postoperative hemorrhage.

\section{Clinical and Radiographic Follow-Up}

At a mean clinical follow-up of 19.1 months (range postoperative to 71 months), 17 patients demonstrated improvement or resolution of their presenting symptoms. These included resolution of intermittent headaches in 17 patients, improvement or complete resolution of sensory complaints in all patients who presented with sensory disturbance, no further episodes of loss of consciousness in 2 patients, and no further hypersomnolence in 1 patient. No patient exhibited symptomatic worsening at last follow-up. Imaging at follow-up in all patients demonstrated complete cyst removal and no evidence of occipital lobe injury.

\section{Case Examples \\ Case 1}

A 27-year-old woman presented with a 3-year history of debilitating intermittent headaches that had prevented her from working as an accountant. Although she had been diagnosed with migraines, her headaches had not improved with medical treatment. In addition, her headaches had a strong positional character, being worse just after standing from a recumbent position. At times, her headaches were associated with bilateral visual blurring but no visual obscurations or blindness. MRI demonstrated a 9-mm pineal cyst. Compression of the tectal plate, admittedly small, was noted on MRI without ventriculomegaly or overt aqueductal obstruction (Fig. 1 left). Given the intermittent, positional features of her headaches accompanied by visual symptoms, surgery was offered as a possible treatment, with the understanding that her symptoms might not be attributable to the pineal cyst.

The patient underwent an uncomplicated interhemispheric occipital-transtentorial approach with gross-total resection of the cyst (Fig. 1 right). Following surgery the patient's headaches resolved, and she remains headache free at last follow-up, 2 years after surgery.

\section{Case 2}

A 28-year-old woman presented with a 3-year history of increasing fatigue and excessive sleep duration. She was diagnosed with idiopathic hypersomnolence syndrome after sleep latency testing, and multiple electroencephalography studies ruled out an underlying seizure disorder. Her hypersomnolence did not improve with modafinil use. At that time, she had been sleeping up to 20 hours per day. In addition to her primary complaint of excessive sleeping, she did report occasional episodic headaches.

MRI demonstrated a 12-mm pineal cyst without ventriculomegaly (Fig. 2 left). Although definite attribution of the patient's symptoms to the lesion was not possible, given her intractable symptoms, the lack of a clear cause, and a theoretical association between pineal lesions and sleep disturbance, she was offered resection.

The patient underwent an uncomplicated resection with gross-total resection of the pineal cyst. Three months

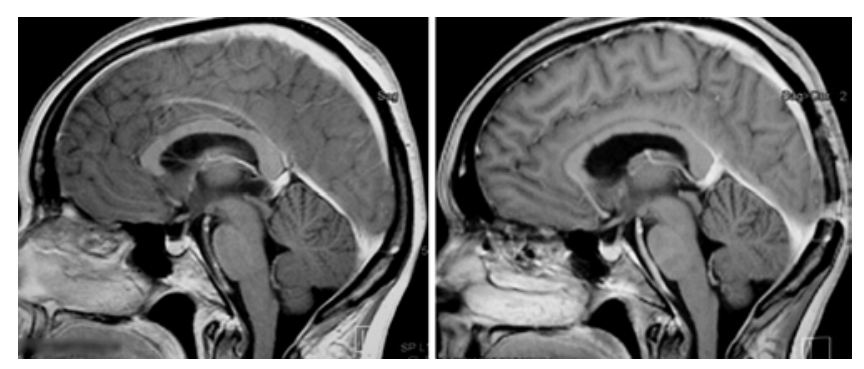

FIG. 1. This 27-year-old woman presented with severe intermittent headaches. The preoperative sagittal T1-weighted MRI (left) demonstrates a 9-mm pineal cyst with mild compression of the tectal plate but no overt aqueductal stenosis and no ventriculomegaly. The postoperative sagittal sequence (right) demonstrates complete cyst resection. Postoperatively, the patient's symptoms resolved entirely.

after surgery she experienced significant improvement of her hypersomnolence. By 8 months after surgery her hypersomnolence had completely resolved. Sleep studies revealed that the duration of sleep posttreatment was 9-10 hours per night. The patient experienced no further fatigue upon awakening. At 8 months her transient postoperative diplopia had resolved and imaging demonstrated no residual or recurrent cyst (Fig. 2 right). Twelve months after surgery, she had a normal sleep pattern.

\section{Discussion}

Pineal cysts are usually asymptomatic, incidental lesions. ${ }^{1-3}$ Although commonly identified during workup for headaches, in the majority of cases, they are not the source of symptoms. ${ }^{1-3,6,8,22}$ In rare cases, large cysts may obstruct the cerebral aqueduct, causing ventriculomegaly or compress the tectum, causing Parinaud's syndrome. . $10,11,15,18,26^{2}$ In our practice we have treated 3 patients who presented with hydrocephalus caused by compression of the aqueduct by a pineal cyst. While these findings are well-accepted indications for surgery, the majority of patients with pineal cysts do not present with these signs, and indications for intervention in these patients is unclear.

Pineal cysts have long been recognized as potentially symptomatic lesions and were first described as a patho-
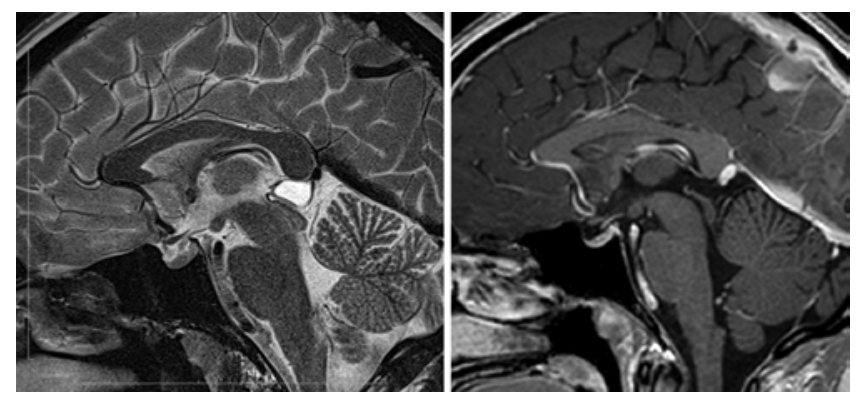

FIG. 2. This 28-year-old woman presented with headaches and an idiopathic hypersomnolence syndrome. Her preoperative T2-weighted sagittal MRI (left) demonstrates a 12-mm pineal cyst with out ventriculomegaly. Her postoperative T1-weighted MRI (right) demonstrates complete cyst resection. Following surgery, her hypersomnolence syndrome resolved. 
logical entity in 1899. ${ }^{4}$ Carr reported a series of 6 large pineal cysts in 1944, 3 of which were symptomatic. ${ }^{5}$ Over the subsequent years, sporadic reports of large symptomatic pineal cysts causing headache, Parinaud's syndrome, long tract signs, and pineal apoplexy appeared in the literature. ${ }^{10,11,17,19,20,25}$ Although rare cases of pineal cysts continued to be reported, in nearly all cases the determination of "symptomatic" relied on signs of ventriculomegaly or Parinaud's syndrome. Klein and Rubinstein reported that among a surgical series of 7 symptomatic pineal cysts ranging in size from 1.0 to $4.5 \mathrm{~cm}, 5$ patients demonstrated improvement following surgery. ${ }^{15}$ Although the majority of patients in this series presented with Parinaud's syndrome, one patient with positional headaches in the absence of Parinaud's syndrome or ventriculomegaly improved following surgery. Wisoff and Epstein reported excellent surgical outcomes with 6 symptomatic pineal cysts, all of which presented with features of Parinaud's syndrome or ventriculomegaly. ${ }^{26}$ More recently, Michielsen and colleagues reported success in surgically treating pineal cysts causing obstructive hydrocephalus, but also noted a lack of improvement in 2 cases without ventriculomegaly. ${ }^{18}$ While these studies all establish large pineal cysts as potentially symptomatic lesions, most reports only included large lesions causing ventriculomegaly or Parinaud's syndrome. For this reason, these studies fail to address the question of how to best manage a patient with a pineal cyst but without overt signs of intracranial hypertension or symptomatic mass effect.

In the current series of patients with pineal cysts without overt hydrocephalus or tectal compression, 17 of 18 patients demonstrated resolution or improvement of their presenting symptoms at last follow-up. The mean cyst diameter of our patients was $1.5 \mathrm{~cm}$, with the smallest cyst measuring $9 \mathrm{~mm}$. These results suggest that the absences of ventriculomegaly and Parinaud's syndrome are not absolute contraindications to surgical intervention. We postulate that despite a small cyst size, these patients may experience intermittent occlusion of their CSF pathways akin to intermittent obstruction observed in patients with colloid cysts.

Recent studies have demonstrated that, with the increased use of routine neuroimaging, more incidental pineal cysts are discovered., ${ }^{1,312,22}$ These studies underscore the fact that the vast majority of pineal cysts are asymptomatic and have a benign natural history. In the largest and most recent study, Al-Holou and colleagues reviewed more than 48,000 adult patients undergoing brain MRI and discovered 478 incidental pineal cysts greater than 5 $\mathrm{mm}$ on MRI. ${ }^{3}$ Although $21 \%$ of their patients presented with headache, no cysts were deemed to be symptomatic and no patient underwent cyst resection. At follow-up, 99\% of cysts remained stable or decreased in size. This study and others demonstrate that the vast majority of pineal cysts are asymptomatic and do not have a propensity to grow. However, because the other authors required the presence of Parinaud's syndrome, focal cranial neuropathies, or obstructive hydrocephalus to classify a cyst as symptomatic, it is possible that symptomatic patients without these signs were not included. Our results suggest that a subset of these patients may indeed benefit from surgery.
It is important to note that our data do not imply that most pineal cysts are symptomatic or that surgery should be offered in many cases. Our series represents a highly selected group of patients with symptoms suggestive of intermittent CSF obstruction for which no other cause was identified after thorough investigations. These data are not inconsistent with large-scale studies demonstrating that most pineal cysts are asymptomatic, even in the presence of headache. Rather, our experience suggests that a small subset of pineal cyst patients without overt symptoms of brainstem compression or ventriculomegaly may benefit from surgery.

Several groups have reported the hypothesis that pineal cysts may cause symptoms from intermittent obstruction of CSF pathways without ventriculomegaly. ${ }^{13,16,26}$ Our series represents the largest experience treating pineal cysts on the principle of intermittent CSF obstruction. Determining whether intermittent CSF obstruction is present may be challenging, and in none of our cases was a definitive association between cyst and symptoms established preoperatively. In selecting patients, our results suggest that episodic positional headaches, paroxysmal headaches associated with bilateral visual blurring or diplopia, and unexplained episodic loss of consciousness may be consistent with intermittent CSF obstruction, although none of these signs is specific. The decision to offer surgery should be based on a combination of clinical symptoms, radiographic findings, and a diagnosis of exclusion and be made only after extensive consultation with and education of the patients. In each case, surgery was offered after clear discussion of the possibility that the symptoms may be unrelated to the lesion.

\section{Limitations}

This study suffers from the well-established shortcomings of a retrospective study. Due to the referral pattern of our practice, the types of pineal cysts encountered by our group represent a selected cohort of patients for whom neurosurgical intervention has already been considered as a possible option by a neurologist. For this reason, significant selection bias prevents our study from assessing the prevalence of intermittent CSF obstruction among all pineal cysts in the general population. Likewise, our study is not designed to estimate what proportion of patients with pineal cysts may benefit from surgery. Given the high prevalence of pineal cysts in the general population, it is likely that the fraction of pineal cyst patients for whom surgical intervention is appropriate remains small. Furthermore, our study did not use quantitative quality of life measures to assess improvement in patient symptoms after intervention. Follow-up studies should focus on better deciphering the degree of improvement with surgery. Nonetheless, our study represents a large cohort of carefully selected patients who demonstrated symptomatic qualitative improvement after cyst removal. Although some patients may have benefited from a placebo effect from surgery, it is unlikely that 18 consecutive patients would all be placebo responders; therefore, the mechanical act of removing an obstruction from the CSF pathways likely has a physiological response in this patient population. 


\section{Conclusions}

Our results suggest that a large cyst size, ventriculomegaly, and Parinaud's syndrome are not the only indications for a pineal cyst to be symptomatic and that a carefully selected cohort of patients without these findings may benefit from cyst resection.

\section{References}

1. Al-Holou WN, Garton HJ, Muraszko KM, Ibrahim M, Maher CO: Prevalence of pineal cysts in children and young adults. Clinical article. J Neurosurg Pediatr 4:230-236, 2009

2. Al-Holou WN, Maher CO, Muraszko KM, Garton HJ: The natural history of pineal cysts in children and young adults. J Neurosurg Pediatr 5:162-166, 2010

3. Al-Holou WN, Terman SW, Kilburg C, Garton HJ, Muraszko KM, Chandler WF, et al: Prevalence and natural history of pineal cysts in adults. J Neurosurg 115:1106-1114, 2011

4. Campbell A: Notes of two cases of dilatation of central cavity or ventricle of the pineal gland. Trans Pathol Soc (Lond) 50:15-18, 1899

5. Carr J: Cystic hydrops of the pineal gland: with a report of six cases. J Nerv Ment Dis 99:552-572, 1944

6. Choy W, Kim W, Spasic M, Voth B, Yew A, Yang I: Pineal cyst: a review of clinical and radiological features. Neurosurg Clin N Am 22:341-351, vii, 2011

7. Costa F, Fornari M, Valla P, Servello D: Symptomatic pineal cyst: case report and review of the literature. Minim Invasive Neurosurg 51:231-233, 2008

8. Di Costanzo A, Tedeschi G, Di Salle F, Golia F, Morrone R, Bonavita V: Pineal cysts: an incidental MRI finding? J Neurol Neurosurg Psychiatry 56:207-208, 1993

9. Evans RW, Peres MF: Headaches and pineal cysts. Headache 50:666-668, 2010

10. Fain JS, Tomlinson FH, Scheithauer BW, Parisi JE, Fletcher GP, Kelly PJ, et al: Symptomatic glial cysts of the pineal gland. J Neurosurg 80:454-460, 1994

11. Fetell MR, Bruce JN, Burke AM, Cross DT, Torres RA, Powers JM, et al: Non-neoplastic pineal cysts. Neurology 41:1034-1040, 1991

12. Golzarian J, Balériaux D, Bank WO, Matos C, Flament-Durand J: Pineal cyst: normal or pathological? Neuroradiology 35:251-253, 1993

13. Gore PA, Gonzalez LF, Rekate HL, Nakaji P: Endoscopic supracerebellar infratentorial approach for pineal cyst resection: technical case report. Neurosurgery 62 (3 Suppl 1):108-109, 2008

14. Hasegawa A, Ohtsubo K, Mori W: Pineal gland in old age; quantitative and qualitative morphological study of $168 \mathrm{hu}-$ man autopsy cases. Brain Res 409:343-349, 1987

15. Klein P, Rubinstein LJ: Benign symptomatic glial cysts of the pineal gland: a report of seven cases and review of the literature. J Neurol Neurosurg Psychiatry 52:991-995, 1989

16. Mandera M, Marcol W, Bierzyńska-Macyszyn G, Kluczewska E: Pineal cysts in childhood. Childs Nerv Syst 19:750-755, 2003

17. Maurer PK, Ecklund J, Parisi JE, Ondra S: Symptomatic pineal cyst: case report. Neurosurgery 27:451-454, 1990

18. Michielsen G, Benoit Y, Baert E, Meire F, Caemaert J: Symptomatic pineal cysts: clinical manifestations and management. Acta Neurochir (Wien) 144:233-242, 2002

19. Oeckler R, Feiden W: Benign symptomatic lesions of the pineal gland. Report of seven cases treated surgically. Acta Neurochir (Wien) 108:40-44, 1991

20. Osborn RE, Deen HG, Kerber CW, Glass RF: A case of hemorrhagic pineal cyst: MR/CT correlation. Neuroradiology 31:187-189, 1989

21. Patel AJ, Fuller GN, Wildrick DM, Sawaya R: Pineal cyst apoplexy: case report and review of the literature. Neurosurgery 57:E1066, 2005

22. Pu Y, Mahankali S, Hou J, Li J, Lancaster JL, Gao JH, et al: High prevalence of pineal cysts in healthy adults demonstrated by high-resolution, noncontrast brain MR imaging. AJNR Am J Neuroradiol 28:1706-1709, 2007

23. Seifert CL, Woeller A, Valet M, Zimmer C, Berthele A, Tölle T, et al: Headaches and pineal cyst: a case-control study. Headache 48:448-452, 2008

24. Tapp E, Huxley M: The histological appearance of the human pineal gland from puberty to old age. J Pathol 108:137144, 1972

25. Vaquero J, Martínez R, Escandón J, Bravo G: Symptomatic glial cysts of the pineal gland. Surg Neurol 30:468-470, 1988

26. Wisoff JH, Epstein F: Surgical management of symptomatic pineal cysts. J Neurosurg 77:896-900, 1992

\section{Author Contributions}

Conception and design: Teo, Wilson. Acquisition of data: Teo, Wilson, Koechlin, Abuhusain, Dlouhy, Gunawardena, NozueOkada. Analysis and interpretation of data: Teo, Wilson, Koechlin, Abuhusain, Dlouhy, Gunawardena, Nozue-Okada. Drafting the article: Teo, Kalani, Wilson, Koechlin, Abuhusain, Dlouhy, Nozue-Okada. Critically revising the article: Teo, Kalani, Wilson, Koechlin, Abuhusain, Dlouhy. Reviewed submitted version of manuscript: Teo, Kalani, Koechlin, Abuhusain. Statistical analysis: Wilson, Koechlin, Gunawardena. Administrative/technical/material support: Gunawardena. Study supervision: Teo.

\section{Correspondence}

Charles Teo, Centre for Minimally Invasive Neurosurgery, Prince of Wales Hospital, Suite 3, Level 7, Barker St., Randwick, Sydney, NSW 2031, Australia. email: Charlie@neuroendoscopy.info. 